\title{
Câncer de pele em trabalhadores rurais: fotoexposição e orientação quanto a fatores de risco
}

Skin cancer in rural workers: photoexposure and guindance on risk factors

Cáncer de piel em trabajadores rurales: foto exposición y orientación sobre factores de riesgo

Recebido: 30/12/2020 | Revisado: 31/12/2020 | Aceito: 04/01/2021 | Publicado: 06/01/2021

\author{
Mainara Meller Dalcin \\ ORCID: https://orcid.org/0000-0001-7457-8858 \\ Sociedade Educacional Três de Maio, Brasil \\ E-mail: mainaramellerdalcin@gmail.com \\ Gabriele Catyana Krause \\ ORCID: https://orcid.org/0000-0001-8340-4534 \\ Sociedade Educacional Três de Maio, Brasil \\ E-mail: gabrielekrause@ setrem.com.br \\ Carlice Maria Scherer \\ ORCID: https://orcid.org/0000-0002-9184-6794 \\ Sociedade Educacional Três de Maio, Brasil \\ E-mail: carlice@ setrem.com.br \\ Silvana Ceolin \\ ORCID: https://orcid.org/0000-0001-6635-5515 \\ Sociedade Educacional Três de Maio, Brasil \\ E-mail: silvanaceolin@gmail.com \\ Gabriel Lautenschleger \\ ORCID: https://orcid.org/0000-0001-7876-130X \\ Universidade Federal de Santa Maria, Brasil \\ E-mail: gabriel.lauten20@gmail.com \\ Marcio Rossato Badke \\ ORCID: https://orcid.org/0000-0002-9459-1715 \\ Universidade Federal de Santa Maria, Brasil \\ E-mail: marciobadke@gmail.com
}

\begin{abstract}
Resumo
O estudo busca determinar o perfil epidemiológico dos trabalhadores rurais moradores do noroeste do estado do Rio Grande do Sul e verificar seu conhecimento sobre proteção e fatores de risco para o desenvolvimento do câncer de pele. Trata-se de um estudo descritivo, exploratório e de caráter quantitativo, realizado com trabalhadores rurais do município de Horizontina. Muitos entrevistados possuem algum tipo de informação e orientação sobre o câncer de pele e sua prevenção, porém, grande parte não utiliza medidas de proteção como roupas adequadas e protetor solar e, se expõe ao sol em horários inadequados. Além disso, possuem histórico de câncer de pele na família, fator influente para o desenvolvimento do mesmo. Ressalta-se que a maioria possui uma propriedade pequena e o serviço é manual, levando-os a uma maior exposição a outros fatores. Nota-se que a exposição solar é prejudicial para a população principalmente devido a exposição cumulativa e a falta de conscientização para o risco de desenvolvimento de câncer de pele. Além disso, essa população ainda é muito afetada pela falta de acessibilidade a educação, como grupos de promoção e prevenção a saúde. Nesse sentido, percebe-se a necessidade de orientações, fortalecendo a importância da prevenção do câncer de pele.
\end{abstract}

Palavras-chave: Câncer de pele; População rural; Prevenção.

\begin{abstract}
The study aims to determine the epidemiological profile of rural workers living in the northwest of the state of Rio Grande do Sul and to verify their knowledge about protection and risk factors for skin cancer development. This is a descriptive, exploratory, and quantitative study, carried out with rural workers in Horizontina city. Many interviewees have some type of information and guidance on skin cancer and its prevention, however, most of them do not use protective measures such as appropriate clothes and sunscreen and, if exposed to the sun at inappropriate times. In addition, they have a family history of skin cancer, an influential factor for its development. It is noteworthy that most have a small property and the service is manual, leading them to greater exposure to other factors. It is noted that sun exposure is harmful to the population mainly due to cumulative exposure and lack of awareness of the risk of developing skin cancer. In addition, this population is still very affected by the lack of access to education, such as health promotion and prevention groups. In this sense, there is a need for guidance, strengthening the importance of skin cancer prevention.
\end{abstract}


Keywords: Skin Cancer; Rural population; Prevention.

\section{Resumen}

El estudio busca determinar el perfil epidemiológico de los trabajadores rurales que viven en el noroeste del estado de Rio Grande do Sul y verificar su conocimiento acerca de la protección y factores de riesgo para el desarrollo de cáncer de piel. Se trata de un estudio descriptivo, exploratorio y cuantitativo, realizado con trabajadores rurales del municipio de Horizontina. Muchos entrevistados poseen algún tipo de información y orientación sobre el cáncer de piel y su prevención, sin embargo, gran parte no utiliza medidas de protección como ropas adecuadas y protector solar y, se expone al sol en horarios inadecuados. Además de eso, poseen antecedentes familiares de cáncer de piel, factor influyente para su desarrollo. Es de destacar que la mayoría posee una propiedad pequeña y el servicio es manual, lo que los lleva a una mayor exposición a otros factores. Se nota que la exposición solar es perjudicial para la población principalmente debido a la exposición acumulativa y la falta de concientización para el riesgo del desarrollo de cáncer de piel. Por otro lado, esta población aún sigue muy afectada por la falta de acceso a la educación, como los grupos de promoción y prevención a la salud. En este sentido, se percibe la necesidad de orientaciones que refuercen la importancia de la prevención del cáncer de piel.

Palabras clave: Cáncer de piel; Población rural; Prevención.

\section{Introdução}

O câncer é caracterizado como um crescimento desordenado de células, com potencial de propagar-se para outras regiões do corpo, invadindo tecidos e órgãos, em um processo chamado de metástase. Entre os diferentes tipos de carcinoma, destaca-se o câncer de pele, hoje em dia o de maior incidência no Brasil (Instituto Nacional do Câncer [INCA], 2020).

Atualmente as estatísticas em relação a esse tipo de câncer no Brasil chegam a 8.450 casos em 2020. Já no Rio Grande do Sul, tem-se o câncer de pele não melanoma como o mais incidente dentre todas as regiões do país com uma taxa estimada de 158,12 casos para cada 100 mil homens e 119,47 casos para cada 100 mil mulheres, já o câncer de pele melanoma possui uma taxa estimada de 5,26 casos para cada 100 mil homens, e de 4,80 casos para cada 100 mil mulheres (INCA, 2020).

O câncer de pele pode ser dividido, basicamente, em dois tipos câncer de pele melanoma quando tem origem nos melanócitos (células produtoras de pigmentos que dão cor à pele), podendo aparecer em qualquer parte do corpo, pele ou mucosas, sendo em forma de manchas, pintas ou sinais (Brasil, 2020). E câncer de pele não melanoma, subdividido em carcinoma basocelular e carcinoma epidermóide. O carcinoma basocelular frequentemente afeta a região do nariz, locais com bastante exposição ao sol, como no rosto, pescoço, orelha e outros; o carcinoma epidermóide, origina-se nas células epiteliais, presente na pele e na camada escamada das mucosas - esôfago, laringe, boca, canal anal, pulmões, colo uterino e outros (Cezar et al., 2015).

Como outros, o câncer de pele não tem uma causa única podendo ocorrer devido a fatores externos que estão presentes no meio ambiente, ou fatores internos como mutações genéticas. O principal fator de risco associado a doença é a fotoexposição excessiva, principalmente quando esta ocorre sem proteção adequada (Bomfim, Giotto \& Silva, 2018).

A fotoexposição está presente em diferentes profissões, destacando-se os trabalhadores rurais. Hayashide et al. (2010) observaram em seus estudos sobre doenças de pele em trabalhadores rurais, que existe uma grande necessidade de ampliar a medicina preventiva e a promoção de suporte ao atendimento básico de saúde nessa população.

Destaca-se também, que ações como a promoção, prevenção, tratamento e recuperação de saúde, devem ser analisadas conforme o contexto social do paciente, pois na área rural estes possuem características distintas, na sua forma de viver, trabalhar e, nesse sentido, se expõe a ambientes mais propícios a algumas doenças específicas como câncer de pele, intoxicações, entre outras (Moreira et al., 2015).

Silva, Souza e Silva (2015) destacaram em sua pesquisa que, apesar de os entrevistados terem conhecimento sobre os riscos decorrentes à exposição solar de forma inadequada, os mesmos ainda continuam a praticar suas atividades sem proteção adequada durante o período exposto, concluindo que apesar das campanhas de conscientização e o contato direto nas unidades básicas de saúde, os profissionais não devem medir esforços para buscar novas tecnologias que possam chamar mais atenção 
da população, melhorando a orientação para assim reduzir a incidência e a mortalidade relacionada ao câncer de pele em trabalhadores rurais.

Em 2016, a Sociedade Brasileira de Dermatologia (SBD) destacou alguns hábitos da população brasileira, os quais evidenciam a exposição ao sol de forma intencional e sem proteção, aumentando o risco de desenvolver câncer. A SBD destaca que a orientação correta é muito importante, pois especifica para a população os cuidados e comportamentos que devem ser tomados para minimizar os efeitos da exposição (Sociedade Brasileira de Dermatologia [SBD], 2020).

Dessa maneira, torna-se de extrema importância o conhecimento da população em relação aos fatores de risco e medidas de prevenção. Ademais, existem poucos estudos que abordam os aspectos comportamentais em relação a prevenção do câncer de pele, e o nível de conhecimento da população em relação à essas medidas preventivas, o que justifica a importância da realização deste estudo, tendo em vista tanto o aumento no índice de novos casos quanto à falta de conscientização e orientação da população. Nesse sentido, o presente estudo visa determinar o perfil epidemiológico dos trabalhadores rurais moradores do noroeste do estado do Rio Grande do Sul, assim como verificar seu conhecimento sobre proteção e fatores de risco para o desenvolvimento do câncer de pele.

\section{Métodos}

Trata-se de um estudo descritivo, exploratório e de caráter quantitativo, realizado em forma de pesquisa de campo que tem por finalidade, compreender mais sobre o assunto e conhecer a estrutura da localidade, tendo em vista a realidade dos fatos, buscando respostas para problemas, também entender fenômenos que ocorrem nas diversas áreas do saber. A técnica utilizada foi a de entrevista, onde realizou-se a coleta dos dados, o diagnóstico e orientações. O estudo foi realizado com trabalhadores em área rural de um município de Horizontina-RS, localizado no noroeste do estado do Rio Grande do Sul (Pereira et al., 2018).

A população da pesquisa foi constituída de trabalhadores rurais, a seleção amostral utilizou como critérios de inclusão: trabalhadores residentes na área rural do município; idade mínima de 18 anos completos; atuar na agricultura; aceitar fazer parte da pesquisa. Foram excluídos trabalhadores que não se encaixavam nos critérios de inclusão.

Dados da prefeitura municipal atualizados em 2016, descrevem a área da unidade territorial: $229.736 \mathrm{~km}^{2}$, população total: 19.117 , população rural: 3.779 , população urbana: 14.571 , tendo assim como crescimento anual da população: 5,7\%. O número da população rural é 3.779 (Horizontina, 2020), a partir desta população, aplicou-se o cálculo amostral n=N.Z2.p.(1-p) / Z2.p.(1-p) + e2.N-1 (n: amostra calculada, N: população, Z: variável normal, p: real probabilidade do evento, e: erro amostral) $(\mathrm{Geo}, 2017)$ para determinar o número de indivíduos que seriam entrevistados. Considerando um nível de confiança de $90 \%$ e margem de erro de $5 \%$ a amostra foi de 252 pessoas.

As entrevistas foram realizadas no primeiro semestre de 2020 na residência dos entrevistados, foi utilizado um formulário com perguntas objetivas abordando os aspectos do tema prevenção/ exposição aos raios ultravioletas. Durante a entrevista foi realizado a entrega do Termo de Consentimento Livre e Esclarecido. Após a coleta os dados foram analisados pelo uso da estatística descritiva simples, utilizando-se o método de distribuição de frequência e percentual no software Excel. A apresentação da análise dos dados é em forma de gráficos representativos, que abordam as perguntas realizadas durante a busca.

O projeto seguiu as resoluções necessárias, que se refere aos aspectos éticos da pesquisa envolvendo seres humanos, sendo assim, aceito pelo comitê de ética da Universidade de Passo Fundo - RS, aprovado com o número de registro CAAE: 20116019.5.0000.5342, e como número de parecer 3.642.342. 


\section{Resultados}

Dos 252 entrevistados, a faixa etária mais prevalente encontrada na população estudada é maior de 50 anos de idade (n=167), já na faixa de 18 a 30 anos, teve-se a participação de 31 pessoas e na faixa de 31 a 49 anos, 54 entrevistados. Destacase uma participação maior do sexo feminino ( $\mathrm{n}=141)$ em relação ao sexo masculino (n=111).

O nível de escolaridade da população rural do município de Horizontina e a orientação quanto a fatores de proteção de câncer de pele são apresentadas na figura 1. Dentre os entrevistados, $54 \%$ possuem baixo nível de escolaridade, com ensino fundamental incompleto; $6 \%$ possuem o ensino fundamental completo; $8 \%$ ensino médio incompleto, $25 \%$ ensino médio completo, $6 \%$ ensino superior completo e 1\% possui o superior incompleto (Figura 1A). Quanto aos dados sobre orientação a prevenção ao câncer de pele a maioria (66\%) afirmou já ter recebido alguma orientação por meio de agentes de saúde, palestras ou até mesmo nas redes sociais e a minoria (34\%) diz não ter recebido nenhuma orientação (Figura 1B).

Figura 1. Nível de escolaridade da população rural de Horizontina (1A) e orientação quanto a fatores de proteção (1B).

A

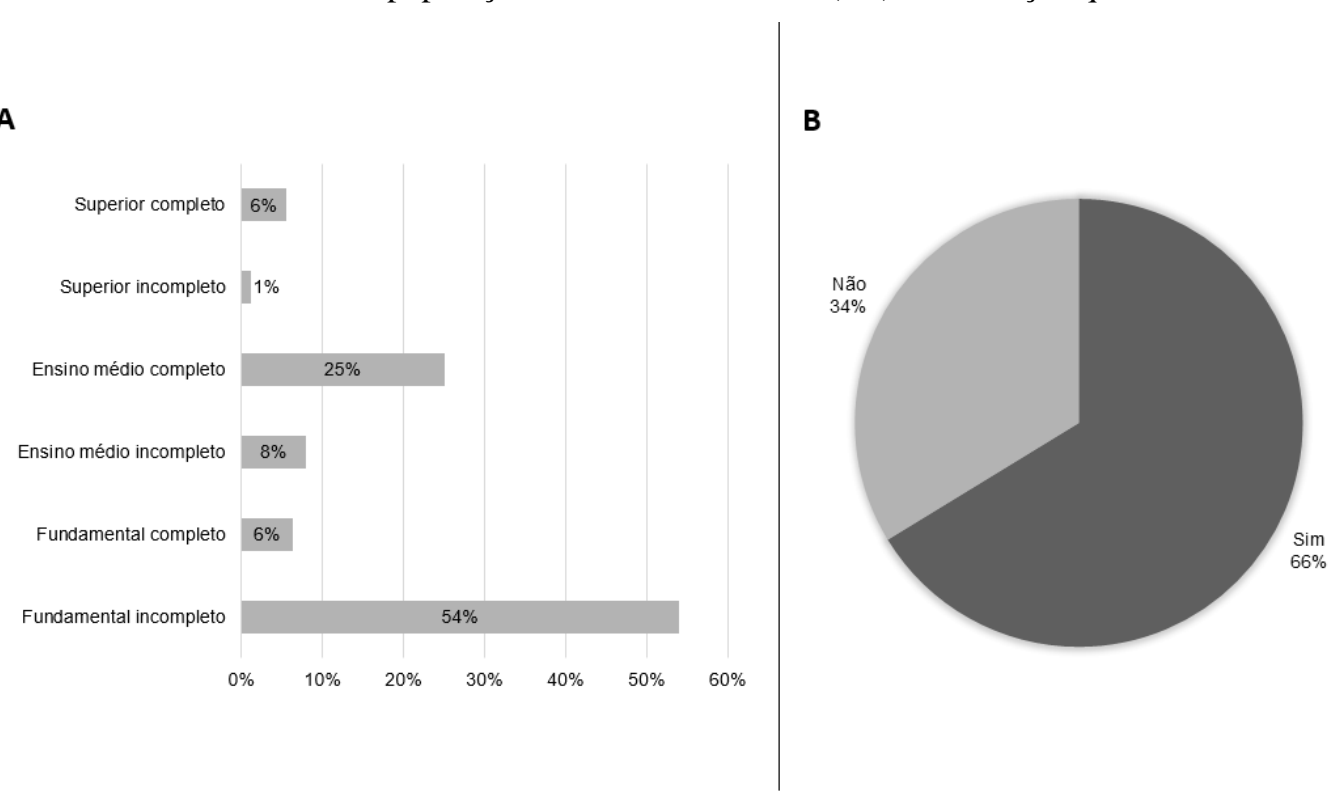

Fonte: Autores.

O tempo de exposição solar, o uso de protetor solar e de roupas adequadas são apresentados na figura 2. A maioria dos entrevistados declararam que ficam pouco tempo expostos ao sol, sendo $42 \%$ de 1 a 2 horas, $19 \%$ de 2 a 3 horas e $39 \%$ mais de 3 horas (Figura 2A). Quando questionados sobre o uso de protetor solar, 42\% diz utilizar regularmente protetor solar; $38 \%$ nunca faz o uso; e $20 \%$ faz o uso diariamente (Figura 2B). Sobre o uso de roupas adequadas, como boné, chapéu, camiseta manga longa e óculos de sol, 69\% relata fazer o uso e 31\% não utiliza nenhum adereço como proteção (Figura 2C). 
Figura 2. Tempo de exposição solar (2A) / Uso de protetor solar (2B) / Uso de roupas adequadas (2C).

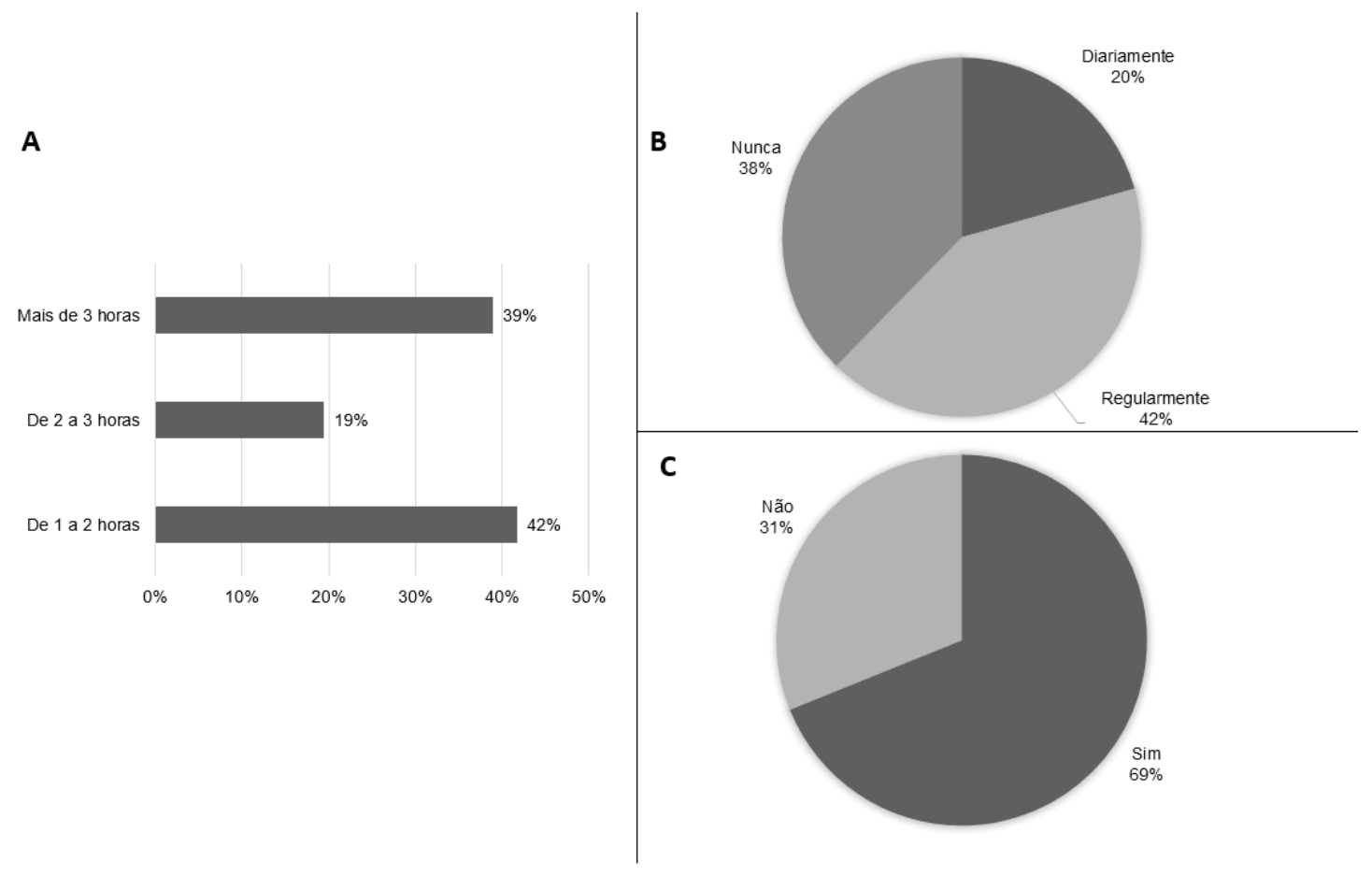

Fonte: Autores.

Durante a pesquisa foram coletados dados sobre o tamanho das propriedades e também dados sobre o uso de maquinário ou serviço manual. Podemos perceber que quanto ao tamanho da propriedade, 64\% dos entrevistados consideram como pequena; $33 \%$ considera como média; e 3\% avalia como grande. Referindo o serviço utilizado na propriedade, $75 \%$ dos entrevistados relatou que realiza serviço manual, o restante $25 \%$ descreve que faz o uso de maquinário.

O histórico de câncer e a área corporal afetada são apresentados na figura 3. Em relação ao histórico de câncer de pele na família, 68\% não possuem nenhum caso em parentesco, mas 32\% relata ter casos da doença na família (Figura 3A). Destes, o câncer de pele encontrava-se em 95\% dos casos em área exposta, como cabeça, pescoço, face, orelha, mãos, antebraços e ombros; e outros 5\% em lugares pouco expostos (Figura 3B). 
Figura 3. Histórico de Câncer na família (3A) / Área corporal afetada (3B).

A

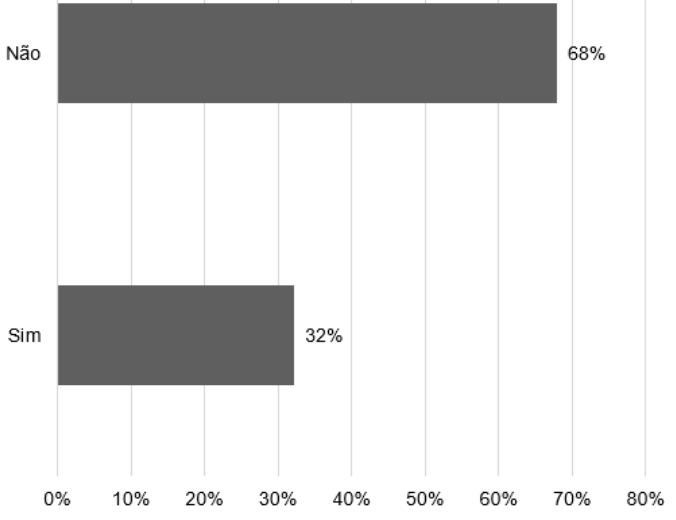

B



Fonte: Autores.

\section{Discussão}

O município estudado tem como principal atividade a produção agrícola, onde predominam micro, pequenas e médias propriedades, com uma média de 10 hectares por bloco de produtor rural. São cultivados soja, milho e trigo, leite, gado de corte, e ainda alguns produtores trabalham com a cultura de suínos e aves, que servem tanto para venda no município quanto para consumo próprio (Jagnow, Jurack \& Stoffel, 2012).

Durante a pesquisa, destacou-se uma prevalência maior de uma população com faixa etária superior a 50 anos (n=167), isso deve-se ao fato de que, possivelmente estes são os responsáveis pelo sustento das famílias, e os mais jovens estão normalmente, envolvidos com outros afazeres como estudo ou até mesmo algum serviço fora da agricultura familiar. Um estudo de Fonseca, Silva e Barbosa (2016) demonstrou que o câncer de pele ocupacional pode ocorrer em indivíduos em idade mais avançada, ressaltando que a exposição cumulativa aos raios ultravioletas é um fator de risco para o desenvolvimento do câncer de pele, tais dados aumentam a importância do presente estudo visto que a população encontrada já está em uma faixa etária mais propensa, devido a exposição cumulativa.

A presença maior de participantes do sexo feminino, justifica-se pelo horário em que foi realizada as entrevistas, entre as 13:00-18:00 horas, sendo possivelmente o horário em que os homens estão trabalhando na propriedade e não se encontravam na residência. Há muitos anos a divisão sexual do trabalho na agricultura familiar vem sendo discutida, Silva, Arantes e Ferreira (2012) afirmam que as tarefas domésticas são assumidas desde cedo pelas mulheres, pois na nossa sociedade o trabalho delas é separado do trabalho dos homens, sendo assim a mulher fica responsável pela limpeza doméstica enquanto o homem com o "trabalho pesado", o que condiz com os resultados encontrados.

Por se tratar de um pequeno munícipio com localidades de difícil acesso, sem escolas próximas e com histórico de falta de transporte público, a maioria dos entrevistados possui ensino fundamental incompleto. Outra questão a ser considerada é a presença dessa população na agricultura desde criança, auxiliando no sustento da família, o que muitas vezes, resulta em abandono aos estudos. Vargas e Ferreira (2020) também afirmam que um dos problemas no campo hoje, é a falta de políticas públicas voltadas ao desenvolvimento e melhoria da qualidade de vida das pessoas que lá vivem e trabalham, ressaltam ainda 
sobre a proposta de Educação Básica do Campo, pois o principal problema para estes é o acesso à escola, desta forma, os jovens e adultos que ali moram, deixam o estudo de lado e continuam a seguir na agricultura familiar.

Outro ponto é que grande parte dos entrevistados relata que por estarem ficando mais velhos, acabam arrendando a terra para se poupar do serviço pesado, o que resulta para eles, em uma pequena propriedade pela qual são responsáveis. Nesse sentido, o serviço manual (75\%) acaba sendo mais viável, e por isso, aparece na maioria da população do estudo. Esta atividade manual se relaciona a uma maior exposição a fatores que podem causar o câncer, ressaltando os fatores externos ou ambientais, pois o agricultor estará exposto a vários agentes, como venenos e agrotóxicos (Chagas, Guimarães \& Boccolini, 2013).

Os fatores externos citados acima, podem ser percebidos quando há visitas domiciliares por parte do profissional de Enfermagem do município, pois este profissional deve avaliar as características culturais, ambientais, educacionais e socioeconômicas do ambiente em que a população vive. Além disso, também é de sua responsabilidade a prevenção e promoção da saúde desta população, que por muitas vezes, acaba sendo excluída de vários processos, como a escolaridade, já citada anteriormente (Schawrtz, Lange \& Meincke, 2001).

Durante a estratificação dos dados, observou-se que apesar dos entrevistados possuírem a baixa escolaridade, os mesmos possuem orientação quanto a prevenção do câncer de pele (66\%), segundo eles, essas informações chegam através de agentes de saúde e grupos de orientação organizados pelas mesmas, assim como por enfermeiras da unidade responsável pela localidade, outra fonte de informação relatada é o compartilhamento de informações em redes sociais.

No entanto, apesar da maioria dos entrevistados possuírem conhecimento sobre o risco de câncer de pele, observa-se que uma grande parte ainda se expõe de maneira inadequada, permanecendo ao sol por mais de 3 horas (39\%). Destaca-se ainda o relato de que, em períodos de safra, essa exposição tende a ser muito maior, podendo durar o dia todo. Esses dados corroboram com o estudo de Silva et al. (2015) que identificou que 44\% dos entrevistados se expõem 7 horas por dia ao sol.

Sabendo que a exposição solar é uma condição inerente da profissão, é de extrema importância o uso de proteção. A indicação, tanto do INCA quanto da SBD, é de evitar a exposição ao sol no período das 10h às 16h, caso seja inevitável a exposição neste período, como é o caso dos agricultores, o indicado é fazer o uso de protetor solar, roupas e adereços adequados (INCA, 2020; SBD, 2020). Recomendação que não condiz com os dados encontrados no estudo, pois somente $42 \%$ dos entrevistados faz uso do protetor solar regularmente.

Outra medida de proteção avaliada, foi a utilização de roupas e adereços como camiseta manga longa, boné, e óculos de sol, nesse sentido, grande parte dos entrevistados (69\%), relatou que fazem o uso da maioria dos adereços, enquanto outros (31\%) optam por não utilizar, ou faz o uso apenas de camiseta manga longa, ou boné, ou óculos. Ainda em estudo de Faria, Rocha e Ribeiro (2019), os resultados são os mesmos em relação as formas de prevenção a exposição aos raios ultravioletas, onde a maioria opta em utilizar camiseta manga longa e outros adereços como forma de prevenção. Considerando que os danos provocados pelo abuso da exposição solar são cumulativos, carece ressaltar que os cuidados devem ser tomados desde a primeira infância. Como já citado, cuidados como o uso de chapéu, guarda-sóis, óculos escuros e camiseta manga longa, são de extrema importância para a prevenção de lesões de pele (INCA, 2020).

A hereditariedade desenvolve um papel central no desenvolvimento do câncer de pele, deste modo, quando há relatos da doença na família, familiares devem se submeter a exames preventivos regularmente (Costa, 2012). Quando questionados sobre o histórico de câncer familiar, muitos dos entrevistados relatam não ter casos na família (68\%), mas ainda são vários os que possuem a doença entre os familiares (32\%). Em estudo, Carvalho, Cunha, Giugliani e Ashton-Prolla (2004) identificaram que o fator hereditário no Brasil é um risco significante em pacientes com melanoma, pois são vários os pacientes que possuem história familiar de múltiplos melanomas, aumentando a suscetibilidade hereditária de desenvolvimento do câncer. 


\section{Considerações Finais}

De maneira geral, percebe-se que a maioria dos participantes do estudo possuem uma baixa escolaridade - poucos possuem o ensino médio completo - apesar disto, muitos possuem algum tipo de informação e orientação sobre o câncer de pele e sua prevenção. Contudo, mesmo com conhecimento sobre os riscos da doença, são vários os que costumam passar o dia exposto ao sol e nem sempre utilizando roupas adequadas e protetor solar. Nesse sentido, percebe-se a necessidade de uma orientação ainda maior para os entrevistados, pois mesmo tendo conhecimento, acabam passando longos períodos expostos ao sol ocasionando um dano cumulativo, gerando um alto risco de desenvolver a doença, além disso, muitos possuem fator hereditário relacionado ao câncer de pele.

Deve-se destacar também a questão do tamanho das propriedades, a maioria relata possuir uma pequena propriedade, onde o serviço realizado, geralmente, é de forma manual, o que contribui para a exposição solar cumulativa, como para outros fatores de risco para o câncer de pele e outras doenças, como uma exposição exacerbada aos agrotóxicos.

No decorrer das entrevistas observou-se os costumes de cada um, e nota-se que muitos não acreditam na prevenção em relação ao câncer de pele, relatando que desde pequenos já estavam expostos a todos os fatores citados e nunca houve algum problema de saúde. Com o intuito de orientar e ressaltar a importância dos cuidados, houve a entrega, durante a entrevista, de um folder ilustrativo com uma breve explicação sobre as principais medidas de prevenção, e como identificar sinais ou manchas na pele que podem indicar a presença de alguma doença. Salientou-se também, sobre a importância dos exames de rotina, e cuidados maiores para os que já estavam em tratamento devido algum problema de pele.

Sabe-se que cada população tem características únicas, no ambiente rural não é diferente, cada família possui sua cultura, funcionamento, limitações, atitudes e uma dinâmica familiar, onde a estratégia da saúde da família só poderá compreender quando presenciar. Assim, destaca-se a importância da realização de visitas domiciliares, por parte da Enfermagem, com o intuito de fortalecer o vínculo da população rural com a saúde, incentivando-os a buscar meios de prevenção e promoção, contribuindo para a qualidade de vida dessas famílias.

Diante disso, ressalta-se que este estudo conseguiu alcançar resultados positivos em relação a avaliação e troca de conhecimentos sobre o assunto, identificando que a população carece de grupos de orientação, ou atividades que possam fortalecer a importância da prevenção de doenças. Acredita-se que este estudo servirá como uma base para que outros Enfermeiros possam buscar um aprofundamento no assunto, e medidas possam ser pensadas e executadas para a prevenção dessa neoplasia. Algumas sugestões dos autores para próximos estudos idênticos, é que busquem um maior detalhamento durante a coleta dos dados, como por exemplo observar outros aspectos relacionados a neoplasia e que possam vir a desenvolve-la, também que busquem uma maior abrangência, tendo resultados ainda mais significativos.

Além disso, o estudo é um dos primeiros artigos da região que buscam como foco a saúde do trabalhador rural, algo que por muitas vezes é deixado de lado, considera-se relevante seu foco, sabendo que a região estudada é uma área onde predomina o trabalho rural. Destaca-se que o câncer de pele é uma questão de saúde pública nacional e mundial, reforçando assim, a necessidade constante de estudos que busquem explorar a promoção e prevenção a saúde da população.

\section{Referências}

Bomfim, S., Giotto, A., \& Silva, A. (2018). Câncer de pele: conhecendo e prevenindo a população. Revisa 7(3), 255-259. https://doi.org/10.3623/revisa.v

Carvalho, C. A., Cunha, M. E., Giugliani, R., Bakos, L., \& Ashton-Prolla, P. (2004). Melanoma hereditário: prevalência de fatores de risco em um grupo de pacientes no Sul do Brasil. Anais Brasileiros de Dermatologia, 79(1), 53-60. https://doi.org/10.1590/S0365-05962004000100006

Cezar-Vaz, M. R., Bonow, C. A., Piexak, D. R., Kowalczyk, S., Vaz, J. C., \& Borges, A. M. (2015). Câncer de pele em trabalhadores rurais: conhecimento e intervenção de enfermagem. Revista da Escola de Enfermagem da USP, 49(4), 0564-0571. https://dx.doi.org/10.1590/S0080-623420150000400005 
Chagas, C. C., Guimarães, R. M., \& Boccolini, P. M. M. (2013). Câncer relacionado ao trabalho: uma revisão sistemática. Cadernos Saúde Coletiva, 21(2), 209-223. https://dx.doi.org/10.1590/S1414-462X2013000200017

Costa, C. S. (2012). Epidemiologia do Câncer de pele no Brasil e evidências sobre sua prevenção. Diagn Tratamento. 17(4):206-8.: https://pdfs.semanticscholar.org/8ad9/261ade0b934e017bc0ad6e1e87fba84dd7d1.pdf

Faria, M. B., Rocha, J. P., \& Ribeiro, M. C. S. (2019). Câncer de pele, fotoexposição e fatores de risco: avaliação de hábitos e conhecimentos de moradores rurais de Orizânia, Minas Gerais. SAPIENS - Revista De divulgação Científica, 1(1). https://revista.uemg.br/index.php/sps/article/view/3436

Geo, S. (2017). Cálculo Amostral. Prática Clínica - Cálculo Amostral. https://praticaclinica.com.br/anexos/ccolaborativa-calculo-amostral/ccolaborativacalculo-amostral.php.

Hayashide, J. M., Minnicelli, R. S., Oliveira, O. A. C. de, Sumita, J. M., Suzuki, N. M., Zambianco, C. A., Framil, V. M. de S., \& Morrone, L. C. (2010). Doenças de pele entre trabalhadores rurais expostos a radiação solar. Estudo integrado entre as áreas de Medicina do trabalho e Dermatologia. Revista Brasileira de Medicina Do Trabalho, 8(2), 97-104.

Horizontina. (2020). Dados demográficos do Município de Horizontina. Portal do Cidadão. http://horizontina.atende.net/

Instituto Nacional do Câncer. (2020). Câncer de Pele não melanoma. Ministério da Saúde. Brasília - DF. https://www.inca.gov.br/tipos-de-cancer/cancer-depele-nao-melanoma

Instituto Nacional do Câncer. (2020b). Como se prevenir do Câncer de Pele no Brasil. Ministério da Saúde. Brasília - DF. https://www.inca.gov.br/causas-eprevencao/como-prevenir-o-cancer

Instituto Nacional do Câncer. (2020c). Estimativa 2020. Ministério da Saúde. Brasília - DF. https://www.inca.gov.br/estimativa/taxas-brutas/pele-naomelanoma

Jagnow, G. E. B., Jurack, I. I., \&Stoffel, J. (2012). Potencialidades do Município de Horizontina. Faculdade Horizontina - FAHOR. 1-20. https://www.fahor.com.br/publicacoes/jopec/2012/POTENCIALIDADES_DO_MUNICIPIO_DE_HORIZONTINA.pdf

Ministério da Saúde. (2020). ABC do câncer: Abordagens Básicas para o Controle do câncer. Inca Rio de Janeiro. https://edisciplinas.usp.br/pluginfile.php/4279235/mod_resource/content/1/abc_do_cancer.pdf

Moreira, J. P. L., Oliveira, B. L. C. A., Muzi, C. D., Cunha, C. L. F., Brito, A. S., \& Luiz, R. R. (2015). A saúde dos trabalhadores da atividade rural no Brasil. Cadernos de Saúde Pública, 31(8), 1698-1708. https://dx.doi.org/10.1590/0102-311X00105114

Pereira, A. S. et al. (2018). Metodologia da pesquisa científica. Ed. https://repositorio.ufsm.br/bitstream/handle/1/15824/Lic_Computacao_Metodologia-Pesquisa-Cientifica.pdf?sequence=1

Schwartz, E., Lange, C., \& Meincke, S. (2001). A enfermagem e os cuidados à saúde da família rural. Família, Saúde e Desenvolvimento, 3(1). doi:http://dx.doi.org/10.5380/fsd.v3i1.4946

Sena, J. S., Girão, R. J. S., Carvalho, S. M. F., Tavares, R. M., Fonseca, F. L. A., Silva, P. B. A., \& Barbosa, M. C. F. P. (2016). Occupational skin cancer: Systematic review. Revista da Associação Médica Brasileira, 62(3), 280-286. https://dx.doi.org/10.1590/1806-9282.62.03.280

Silva, A. C. B. S., Oliveira, G. S., Quental, O. B., Moreira, R. L. S. F., Araújo, W. A., \& Feitosa, A. N. A. (2015). Câncer de pele: conhecimento dos Trabalhadores Rurais do Cariri Cearense. Rev. Interdisciplinar em Saúde, Cajazeiras - CE. 2 (2): 234-249. http://interdisciplinaremsaude.com.br/Volume_4/Trabalho_05.pdf

Silva, C., Arantes R., Ferreira, V. (2012). Nosso Trabalho Sustenta o Mundo. Recife - PE. SOS Corpo. https://soscorpo.org/wp-content/uploads/Nossotrabalho-sustenta-o-mundo-2013-2a-edi\%C3\%A7\%C3\%A3o.pdf

Silva, L. N. M., Souza, C. N. S., Silva, M. C. D. (2015). Medidas de Prevenção contra Câncer de Pele em Trabalhadores Rurais: Contribuição de Enfermagem. 1-10. http://www.fiepbulletin.net/index.php/fiepbulletin/article/viewFile/86.a1.156/12222

Sociedade Brasileira de Dermatologia. (2020). Câncer de Pele. https://www.sbd.org.br/dermatologia/pele/doencas-e-problemas/cancer-da-pele/64/

Sociedade Brasileira de Dermatologia. (2020b). Como prevenir o câncer de pele. Câncer de Pele. http://www.sbd.org.br/dezembroLaranja/noticias/comoprevenir/

Vargas, R. C., Ferreira, L. (2020). A Agricultura Familiar e a Educação no Campo. Universidade Federal do Paraná. 1-11. https://hdl.handle.net/1884/38665 